\title{
Ataxia Severity Correlates with White Matter Degeneration in Spinocerebellar Ataxia Type 7
}

\author{
(D)C.R. Hernandez-Castillo, (DI. Vaca-Palomares, (D) F. Barrios, (D). Martinez, (D) M.-C. Boll, and (D). Fernandez-Ruiz
}

\begin{abstract}
BACKGROUND AND PURPOSE: There is a scarcity of information on the effect of white matter degeneration in patients with spinocerebellar ataxia type 7. Therefore, we investigated the WM integrity in a large group of patients with spinocerebellar ataxia type 7 by using Tract-Based Spatial Statistics.
\end{abstract}

MATERIALS AND METHODS: Thirty-three patients with a molecular diagnosis of spinocerebellar ataxia type 7 and their age- and sex-matched healthy controls participated in this study. The patients' ataxia severity was evaluated with the Scale for the Assessment and Rating of Ataxia. Voxelwise analyses of diffusion metrics, including fractional anisotropy and mean diffusivity, were performed with Tract-Based Spatial Statistics. The correlation between WM abnormalities and ataxia severity was then calculated.

RESULTS: Tract-Based Spatial Statistics analysis revealed WM abnormalities in the cerebellum and the cerebellar peduncles, as well as in other major cortical and subcortical pathways. Further analysis between the Scale for the Assessment and Rating of Ataxia score and WM mean diffusivity showed significant associations only in key areas related to motor control and visuospatial processing, including the cerebellar WM, the middle occipital WM, the superior cerebellar peduncle, and bilateral anterior thalamic radiation. No significant associations between fractional anisotropy and the Scale for the Assessment and Rating of Ataxia were found.

CONCLUSIONS: These results suggest a significant contribution of local cerebellar and cerebellar-midbrain connections to ataxic impairment in spinocerebellar ataxia type 7. The results also suggest an involvement of cortical WM abnormalities including tracts within the occipital and frontal cortices. These findings contribute to a more comprehensive view of the clinical impact of the white matter degeneration in spinocerebellar ataxia type 7.

ABBREVIATIONS: FA = fractional anisotropy; $\mathrm{MD}=$ mean diffusivity; SARA = Scale for the Assessment and Rating of Ataxia; $\mathrm{SCA}=$ spinocerebellar ataxia; $\mathrm{SCA} 7=$ spinocerebellar ataxia type 7; TBSS $=$ Tract-Based Spatial Statistics

$\boldsymbol{S}^{\mathrm{p}}$ pinocerebellar ataxia type 7 (SCA7) is an autosomal dominant cerebellar ataxia caused by a mutation consisting in the expansion of the cytosine-adenine-guanine trinucleotide in the codon region of the chromosome $3 \mathrm{p} 21$, encoding the protein ataxin $7 .^{1}$

Received March 28, 2016; accepted after revision May 26.

From the Consejo Nacional de Ciencia y Tecnologia-Instituto de Neuroetologia (C.R.H.-C.), Universidad Veracruzana, Veracruz, Mexico; Departamento de Fisiologia (I.V.-P., J.F.-R.), Facultad de Medicina, and Instituto de Neurobiologia (F.B.), Universidad Nacional Autonoma de Mexico, Mexico City, Mexico; and Departamento de Neurogenética y Biologia Molecular (L.M.) and Laboratorio de Investigacion Clinica (M.-C.B.), Instituto Nacional de Neurología y Neurocirugía, "Manuel Velasco Suárez," Mexico City, México.

This work was supported by Consejo Nacional de Ciencia y Tecnologia grant 220871 and Universidad Nacional Autonoma de Mexico grant PAPIIT IN214716 to J.F.-R.

Please address correspondence to Juan Fernandez-Ruiz, PhD, Departamento de Fisiología, Facultad de Medicina, Universidad Nacional Autónoma de México, UNAM, Av. Universidad 3000, Coyoacán, Distrito Federal, México, 04510; e-mail: jfr@unam.mx

- Indicates open access to non-subscribers at www.ajnr.org

http://dx.doi.org/10.3174/ajnr.A4903
SCA7 is considered one of the rarest forms of genetic autosomal dominant cerebellar ataxia. ${ }^{2}$ Clinically, SCA7 is characterized by a combination of cerebellar ataxia and macular degeneration and is the only spinocerebellar ataxia that manifests in permanent blindness. ${ }^{3,4}$ Furthermore, patients may eventually develop other neurologic deficits, including loss of manual dexterity, speech dysarthria, dysphagia, and eye movement abnormalities. ${ }^{2}$ A number of neuropathologic studies have documented the anatomic consequences of the neurodegenerative process. These include severe degeneration of the cerebellar cortex and other cortical regions. ${ }^{5,6}$

MR imaging techniques such as diffusion tensor imaging can produce high-resolution structural images of white matter tracts. On the basis of the measurement of water diffusion properties, DTI allows quantifying the tissue microstructure and infering its integrity. ${ }^{7}$ This MR imaging technique also enables mapping of white matter tract changes across the life span and alterations in neurologic disorders, becoming an important tool in the study of neurodegenerative diseases. ${ }^{89}$ Diffusion properties include mean 
diffusivity (MD) (also referred to as apparent diffusion coefficient) and fractional anisotropy (FA). In neurodegenerative diseases including spinocerebellar ataxias (SCAs), both MD and FA have gained widespread acceptance as sensitive indicators for quantifying microstructural damage to white matter, showing the

Table 1: Demographic information of the SCA7 group

\begin{tabular}{|c|c|c|c|c|c|c|}
\hline ID & Sex & $\begin{array}{l}\text { Age } \\
\text { (yr) }\end{array}$ & $\begin{array}{c}\text { Years from } \\
\text { Onset }\end{array}$ & MMSE & $\mathrm{MoCA}$ & SARA \\
\hline P01 & $F$ & 21 & 1 & 26 & NC & 4 \\
\hline P02 & $M$ & 20 & 1 & 29 & NC & 4 \\
\hline P03 & $F$ & 68 & 1 & 24 & NC & 6 \\
\hline P04 & $F$ & 18 & 4 & 27 & NC & 7 \\
\hline P05 & $\mathrm{F}$ & 35 & 6 & 26 & NC & 8.5 \\
\hline P06 & $F$ & 44 & 6 & 26 & 16 & 9 \\
\hline P07 & $F$ & 64 & 13 & 28 & 12 & 9.5 \\
\hline P08 & $M$ & 44 & 4 & 24 & 18 & 11 \\
\hline P09 & $\mathrm{F}$ & 22 & 2 & 26 & NC & 11 \\
\hline P10 & $M$ & 52 & 7 & 26 & 16 & 12 \\
\hline P11 & $\mathrm{F}$ & 45 & 7 & 26 & 21 & 12 \\
\hline $\mathrm{P} 12$ & $M$ & 23 & 3 & 24 & 27 & 12.5 \\
\hline $\mathrm{P} 13$ & $M$ & 47 & 6 & 26 & 14 & 13 \\
\hline P14 & $M$ & 25 & 4 & 26 & 13 & 14 \\
\hline P15 & $M$ & 64 & 10 & 26 & 15 & 14.5 \\
\hline P16 & $M$ & 38 & 13 & 27 & 18 & 14.5 \\
\hline P17 & $\mathrm{F}$ & 43 & 21 & 26 & NC & 15 \\
\hline P18 & $M$ & 35 & 14 & 26 & 9 & 16 \\
\hline P19 & $M$ & 60 & 6 & 26 & 13 & 16 \\
\hline P20 & $M$ & 30 & 6 & 25 & 12 & 16 \\
\hline P21 & $M$ & 34 & 10 & 28 & NC & 17 \\
\hline P22 & $M$ & 30 & 5 & 26 & NC & 17 \\
\hline $\mathrm{P} 23$ & $\mathrm{~F}$ & 32 & 6 & 27 & NC & 19 \\
\hline P24 & $M$ & 18 & 8 & 26 & 16 & 19.5 \\
\hline P25 & $\mathrm{F}$ & 40 & 13 & 25 & NC & 23 \\
\hline P26 & $M$ & 54 & 6 & 25 & NC & 24 \\
\hline P27 & $M$ & 61 & 7 & 22 & NC & 25 \\
\hline P28 & $M$ & 29 & 7 & 21 & 17 & 25 \\
\hline P29 & $\mathrm{F}$ & 40 & 21 & 22 & 18 & 27 \\
\hline P30 & $\mathrm{F}$ & 39 & 23 & 25 & 18 & 27 \\
\hline P31 & $\mathrm{F}$ & 42 & 17 & 23 & 15 & 29.5 \\
\hline P32 & $M$ & 19 & 7 & 22 & 10 & 29.5 \\
\hline P33 & $\mathrm{F}$ & 51 & 8 & 26 & 18 & 30 \\
\hline
\end{tabular}

Note:-MMSE indicates Mini-Mental State Examination; MoCA, Montreal Cognitive Assessment; NC, not completed; ID, identification. associations between the white matter integrity and behavioral impairment in some cases. ${ }^{6,10-13}$ Among several studies using diffusion measurements, only a preliminary study has explored the white matter integrity in a small group of patients with SCA7, ${ }^{6}$ reporting significant FA decreases that correlate with the number of years passed from the onset of symptoms. However, the contribution of the degeneration of projection fibers to the patients' impairments was not explored, possibly due to the small number of subjects. To test this hypothesis, we explored the relationship between ataxia severity and WM metrics that result from the statistical analysis of diffusion tensor imaging in a larger cohort of patients with SCA7.

\section{MATERIALS AND METHODS \\ Subjects}

Thirty-three patients with a molecular diagnosis of SCA7 were invited to participate in this study (15 right-handed women; mean age, $39.0 \pm 14.6$ years). The Scale for the Assessment and Rating of Ataxia (SARA) ${ }^{14}$ was used as a semiquantitative valuation of movement impairment, comprising 8 items related to gait, stance, sitting, speech, finger-chase test, nose-finger test, fast alternating movements, and the heel-shin test. ${ }^{15}$ Extended information about the SCA7 group can be found in Table 1. Thirtythree age- and sex-matched controls (15 right-handed women; mean age, $41.7 \pm 13.8$ years) participated in the study. The control group declared that they did not have any history of neurologic or psychiatric disorders. All procedures were in accordance with the ethical standards of the Declaration of Helsinki of 1975 and the applicable revisions at the time of the investigation. Therefore, the committees on human experimentation of the Universidad Nacional Autónoma de México specifically approved this study. All participants gave their written informed consent before entering the study.

\section{Image Acquisition}

Images were acquired by using a 3T Achieva MR imaging scanner (Philips Healthcare, Best, the Netherlands). The study included the acquisition of a structural high-resolution volume and diffu-

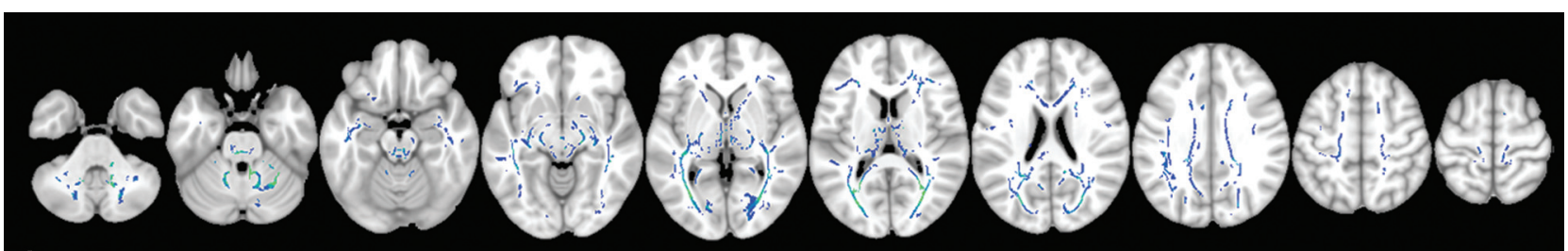

A

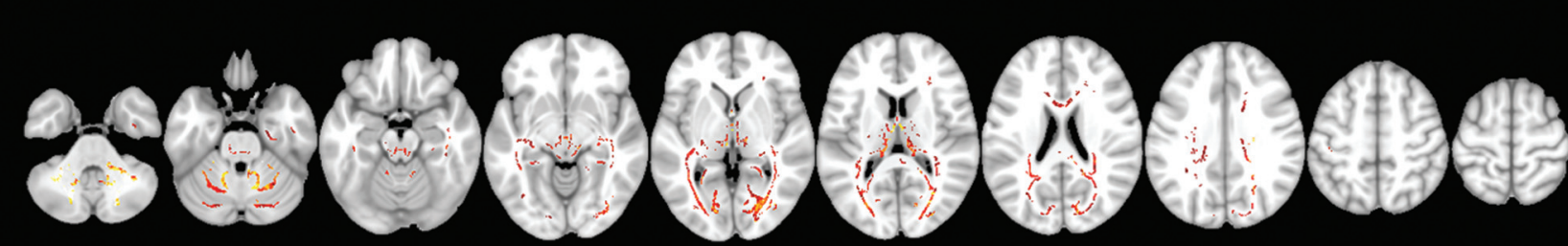

$p<0.05$ corrected

$t=-8$

$-3 \quad 3$

FIG 1. TBSS significant differences in diffusion measurements between patients with SCA7 and healthy controls. A, Fractional anisotropy. $B$, Mean diffusivity. Warm and cold colors indicate an increase and decrease in these measures in patients with SCA7, respectively. 
Table 2: Significant group differences in fractional anisotropy between patients with SCA7 and healthy controls ${ }^{\mathrm{a}}$

\begin{tabular}{lccrrr}
\hline \multicolumn{1}{c}{ Anatomic Region } & Voxels & \multicolumn{1}{c}{ T-Value } & \multicolumn{1}{c}{ X } & \multicolumn{1}{c}{ Y } & \multicolumn{1}{c}{ Z } \\
\hline Right superior cerebellar peduncle & 1797 & 18.9 & 7 & -47 & -28 \\
Left corticospinal tract & 1009 & 11 & -13 & -56 & -28 \\
Left anterior corona radiata & 733 & 6.86 & -26 & 31 & 11 \\
Body of corpus callosum & 327 & 4.04 & -6 & 13 & 22 \\
Right corticospinal tract & 262 & 11 & 13 & -50 & -43 \\
Left uncinate fasciculus & 253 & 6.27 & -21 & 18 & -12 \\
Left superior longitudinal fasciculus & 180 & 5.08 & -10 & 27 & 49 \\
Left inferior longitudinal fasciculus & 177 & 5.75 & -40 & -47 & -13 \\
Right superior longitudinal fasciculus & 162 & 4.53 & 36 & -13 & 34 \\
Left anterior limb of the internal capsule & 134 & 5.07 & -20 & 17 & 5 \\
Left cingulate WM & 131 & 6.36 & -6 & 3 & 34 \\
Left inferior fronto-occipital fasciculus & 121 & 4.89 & -16 & 35 & 33 \\
Left superior longitudinal fasciculus & 114 & 4.75 & -44 & -41 & 13 \\
Right superior longitudinal fasciculus & 112 & 4.22 & 51 & -7 & 23 \\
\hline
\end{tabular}

${ }^{a}$ Coordinates are in millimeters in Montreal Neurological Institute space. Labels are from the Johns Hopkins University white matter atlas.

Table 3: Significant group differences in mean diffusivity between patients with SCA7 and healthy controls ${ }^{a}$

\begin{tabular}{lrrrrr}
\hline \multicolumn{1}{c}{ Anatomic Region } & Voxels & T-Value & \multicolumn{1}{c}{ X } & \multicolumn{1}{c}{ Y } & \multicolumn{1}{c}{ Z } \\
\hline Right middle cerebellar peduncle & 2712 & 11.1 & 26 & -51 & -37 \\
Left middle cerebellar peduncle & 2047 & 12.6 & -26 & -48 & -38 \\
Left anterior thalamic radiation & 911 & 5.12 & -3 & -11 & 11 \\
Right inferior fronto-occipital fasciculus & 855 & 5.19 & 19 & -83 & 16 \\
Left inferior longitudinal fasciculus & 628 & 4.21 & -14 & -81 & 2 \\
Left posterior thalamic radiation & 350 & 3.23 & -35 & -44 & 8 \\
Right inferior longitudinal fasciculus & 182 & 4.39 & 37 & -74 & 2 \\
Right cingulate WM & 141 & 3.59 & 27 & -45 & -2 \\
Right posterior corona radiata & 112 & 5.22 & 29 & -34 & 19 \\
Right anterior thalamic radiation & 108 & 4.16 & 1 & -20 & -10 \\
Splenium of corpus callosum & 104 & 4.91 & 19 & -50 & 22 \\
Left cingulate WM & 101 & 3.58 & -28 & -50 & -1 \\
Left inferior longitudinal fasciculus & 100 & 3.38 & -17 & -77 & 15 \\
\hline
\end{tabular}

${ }^{a}$ Coordinates are in millimeters in Montreal Neurological Institute space. Labels are from the Johns Hopkins University white matter atlas.

sion tensor imaging. For detailed acquisition parameters, please see Hernandez-Castillo et al. ${ }^{11}$

\section{Diffusion Tensor Preprocessing}

The FSL software (http://www.fmrib.ox.ac.uk/fsl) was used to process and analyze the raw DTI data. ${ }^{16}$ First, we corrected the eddy current effects; second, the eddy-corrected diffusionweighted images were spatially normalized by using a rigid-body transformation. Last, the diffusion tensor model was adjusted to generate the fractional anisotropy maps for each participant.

\section{Tract-Based Spatial Statistics}

The statistical analysis was performed in a voxelwise manner by using the standard Tract-Based Spatial Statistics (TBSS; http:// fsl.fmrib.ox.ac.uk/fsl/fslwiki/TBSS) methodology reported elsewhere. ${ }^{17}$ The TBSS procedure had the following steps: All subjects' FA data were aligned into a 1-mm isotropic FA target image in standard space (FMRIB58_FA standard space image; http:// fsl.fmrib.ox.ac.uk/fsl/fslwiki/FMRIB58_FA) by using the FMRIB Nonlinear Registration Tool (FNIRT; http://fsl.fmrib.ox.ac.uk/ fsl/fslwiki/FNIRT).${ }^{18}$ Next, the mean FA image was calculated and thinned to create a mean FA skeleton, which represented the centers of all tracts common to the group. This process had 2 steps: 1 ) averaging the warped FA images, and then 2) thresholding at FA $>0.2$. Each subject's aligned FA data were then projected onto this skeleton. Using the same nonlinear registration derived from the FA analysis, we projected MD data onto the skeleton before voxelwise statistical analysis across subjects. ${ }^{17}$

Differences in DTI (FA, MD) parameters between patients with SCA7 and healthy controls were assessed by using a permutation-based nonparametric independent 2-sample $t$ test (FSL Randomize tool; http://fsl.fmrib.ox.ac.uk/ fsl/fslwiki/Randomise/). ${ }^{19}$ Age was included in the design matrix as a covariate of no interest. Correction for multiple comparisons was assessed by using threshold-free cluster enhancement. ${ }^{20}$ We generated 5000 permutations of the data, producing uncorrected and family-wise errorcorrected statistical maps. Only those voxels surviving this correction at a $P$ value $<.05$ showed a significant group difference. In a second analysis, we explored the correlation between the ataxia severity and WM measurements (FA and MD). We performed a 1-sample $t$ test of the SCA7 group for FA and MD independently, which included the SARA score in the design matrix.

\section{RESULTS}

TBSS group comparison revealed significant FA decreases in patients with SCA7

(Fig $1 A$ and Table 2) in the white matter tracts, including the inferior/middle/superior cerebellar peduncles, the bilateral internal/external capsule, the bilateral corona radiata, the bilateral optical radiation, and the occipital/temporal/ frontal white matter.

TBSS group comparison revealed significant MD increases in patients with SCA7 (Fig $1 B$ and Table 3 ) in the cerebellar WM, including the medial lemniscus, the middle cerebellar peduncle, the optical radiations, the bilateral corona radiata, the posterior limbs of internal capsule, and the corticospinal tract.

We found associations between the patients' SARA scores and mean diffusivity in several abnormal WM tracts. Specifically, we found SARA associations with MD in the right middle occipital $\mathrm{WM}$, stria terminalis, superior cerebellar peduncle, anterior cerebellar WM, superior longitudinal fasciculus, and the anterior thalamic radiation (Fig 2 and Table 4). No significant association was found between the SARA scores and FA.

\section{DISCUSSION}

In this study, we used Tract-Based Spatial Statistics to assess the white matter abnormalities in a group of patients with SCA7 compared with matched healthy controls and the relationship between the white matter integrity and the ataxia severity in the patient group. Our results showed significant differences in FA 


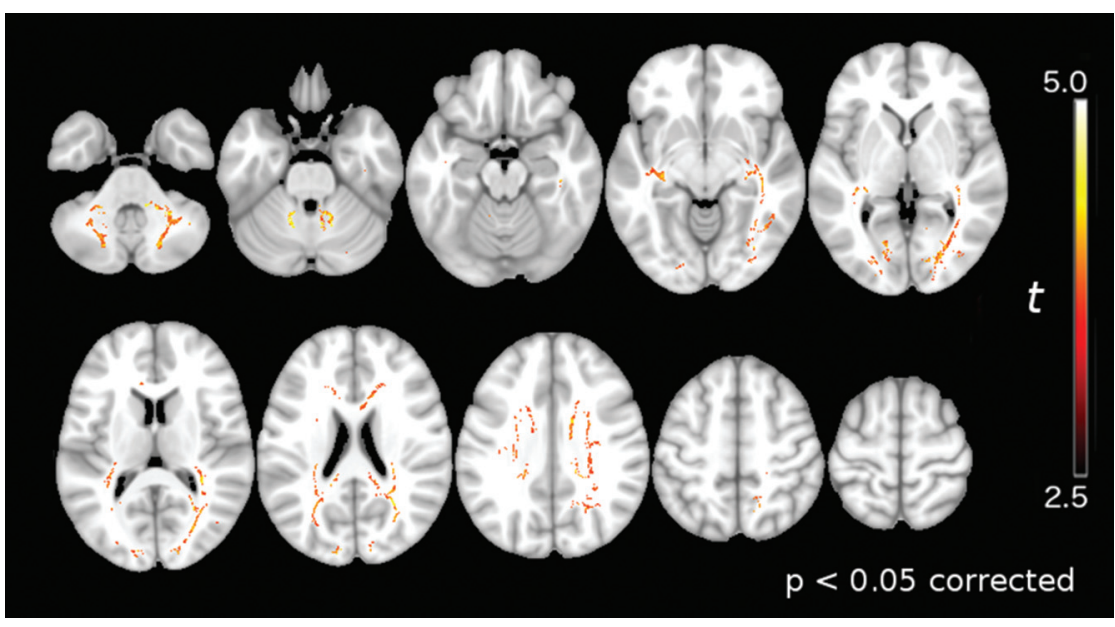

FIG 2. White matter regions where MD correlates with the SARA score in the SCA7 group. Warm colors indicate significant correlations between MD values and SARA scores.

Table 4: White matter regions showing significant correlation between MD and the SARA score in the SCA7 group ${ }^{a}$

\begin{tabular}{lccrrr}
\hline \multicolumn{1}{c}{ Anatomic Region } & Voxels & T-Value & \multicolumn{1}{c}{ X } & \multicolumn{1}{c}{ Y } & \multicolumn{1}{c}{ Z } \\
\hline Right inferior longitudinal fasciculus & 6428 & 6.74 & 40 & -74 & 6 \\
Right superior cerebellar peduncle & 1420 & 9.16 & 7 & -43 & -23 \\
Left cerebellum anterior lobule & 1014 & 7.03 & -14 & -53 & -28 \\
Left inferior fronto-occipital fasciculus & 684 & 7.89 & -19 & -86 & -9 \\
Right anterior thalamic radiation & 239 & 5.92 & 14 & -60 & 49 \\
Right superior longitudinal fasciculus & 158 & 5.54 & 44 & -34 & 34 \\
Left anterior thalamic radiation & 148 & 10.1 & -3 & -9 & 12 \\
\hline
\end{tabular}

${ }^{a}$ Coordinates are in millimeters in Montreal Neurological Institute space. Labels are from the Johns Hopkins University white matter atlas.

and MD when comparing patients with SCA7 with healthy controls and a correlation between the ataxia severity and MD changes in the patient group.

Previous neuropathologic reports have shown loss of myelinated fibers in the cerebellar white matter, corpus callosum, red nucleus capsule, oculomotor nerve, lateral lemniscus, mesencephalic trigeminal tract, abducens nerve, trapezoid body, pontocerebellar fibers, pyramidal tract, internal arcuate and olivocerebellar fibers, cuneate, and gracile fascicles, as well as the spinocerebellar tracts. ${ }^{5,6}$ Our results support those findings but also extend them by showing abnormalities in the occipital $\mathrm{WM}$, the stria terminalis, and the thalamic radiations not previously reported. As expected, the cerebellar WM and the cerebellar peduncles showed a decrease in FA and an increase of $\mathrm{MD}$, implying SCA7-related microstructural changes in the afferent and efferent projections of the cerebellum. The combination of white matter degeneration and gray matter loss in the cerebellum results in a variety of clinical motor impairments, including ataxia and extrapyramidal signs. ${ }^{21}$ In the same way, the degeneration of the thalamic radiations affects the information flow between the cerebellum and the motor and frontal cortices, which might result in the loss of coordination and dexterity in this set of patients.

The most relevant finding of this study is the association of the white matter mean diffusivity and the ataxia severity in this group of patients. The higher correlation between MD and SARA was found in the white matter of the middle occipital gyrus, which is involved in visual and spatial processing. ${ }^{22,23}$ Similarly, the superior longitudinal fasciculus showed significant correlation, which suggests a malfunction in the projections between the occipitalto-frontal cortices, including the premotor areas. A failure of these pathways could affect the planning and action of visuospatial tasks, especially in this disease because it has been reported to show decreases in the functional connectivity between the occipital and motor cortices. ${ }^{24,25}$ Future studies, including neuropsychological evaluation focused on visuospatial performance combined with ophthalmologic data, should be helpful in corroborating this hypothesis.

Other white matter regions showing significant correlation between the MD and SARA score were the superior cerebellar peduncles and the white matter in the anterior cerebellum. On the basis of the distribution of the neuropathologic changes in SCA7, this result was expected. ${ }^{5,6}$ Several reports have shown that lesions in these regions can lead to motor incoordination and loss of movement dexterity. ${ }^{26}$ Both the cerebellar peduncles and the anterior cerebellum have been reported as degenerated in previous MR imaging studies in SCA2. ${ }^{6,24}$ However, in previous studies, no significant correlations were found between the changes in the water diffusion properties and SARA scores, probably due to the small number of participants recruited. ${ }^{6}$

No significant correlations between FA and SARA were found. FA and MD are not equivalent measurements ${ }^{27,28}$; and as expected in our group comparison, FA and MD maps led to different results (Fig 1). In addition to MD and FA, other water diffusion properties such as axial diffusivity and radial diffusivity have been reported in previous studies, including SCA1, SCA2, and Friedreich ataxia. ${ }^{10,29}$ In this work, we focused on the analyses of FA and MD because the other measurements are subcomponents of the apparent diffusion coefficient. Furthermore, several studies of patients with neurodegenerative diseases have suggested that $\mathrm{MD}$ is more useful and sensitive to neurodegeneration than the other measurements. ${ }^{8,9,30}$

\section{CONCLUSIONS}

Our results show that specific changes in the diffusion properties of white matter resulting from the SCA7 mutation are associated with the severity of the ataxia. The distribution of the mean diffusivity abnormalities and its association with SARA scores suggest a disruption of information flow between motor-, visual-, and sensory-integration areas. Overall, these findings contribute to a better understanding of the neural basis of the symptomatology of patients with SCA7.

AJNR Am J Neuroradiol 37:2050-54 Nov 2016 www.ajnr.org 


\section{REFERENCES}

1. Garden GA, La Spada AR. Molecular pathogenesis and cellular pathology of spinocerebellar ataxia type 7 neurodegeneration. Cerebellum 2008;7:138-49 CrossRef Medline

2. Hugosson T, Gränse L, Ponjavic V, et al. Macular dysfunction and morphology in spinocerebellar ataxia type 7 (SCA 7). Ophthalmic Genet 2009;30:1-6 CrossRef Medline

3. Miller RC, Tewari A, Miller JA, et al. Neuro-ophthalmologic features of spinocerebellar ataxia type 7. J Neuroophthalmol 2009;29:180-86 CrossRef Medline

4. Michalik A, Martin JJ, Van Broeckhoven C. Spinocerebellar ataxia type 7 associated with pigmentary retinal dystrophy. Eur J Hum Genet 2004;12:2-15 CrossRef Medline

5. Masciullo M, Modoni A, Pomponi MG, et al. Evidence of white matter involvement in SCA 7. J Neurol 2007;254:536-38 CrossRef Medline

6. Alcauter S, Barrios FA, Díaz R, et al. Gray and white matter alterations in spinocerebellar ataxia type 7: an in vivo DTI and VBM study. Neuroimage 2011;55:1-7 CrossRef Medline

7. Mori S, Zhang J. Principles of diffusion tensor imaging and its applications to basic neuroscience research. Neuron 2006;51:527-39 CrossRef Medline

8. Acosta-Cabronero J, Williams GB, Pengas G, et al. Absolute diffusivities define the landscape of white matter degeneration in Alzheimer's disease. Brain 2010;133:529-39 CrossRef Medline

9. Della Nave R, Ginestroni A, Tessa C, et al. Regional distribution and clinical correlates of white matter structural damage in Huntington disease: a tract-based spatial statistics study. AJNR Am J Neuroradiol 2010;31:1675-81 CrossRef Medline

10. Della Nave R, Ginestroni A, Tessa C, et al. Brain white matter damage in SCA1 and SCA2: an in vivo study using voxel-based morphometry, histogram analysis of mean diffusivity and tract-based spatial statistics. Neuroimage 2008;43:10-19 CrossRef Medline

11. Hernandez-Castillo CR, Galvez V, Mercadillo R, et al. Extensive white matter alterations and its correlations with ataxia severity in SCA 2 patients. PLoS One 2015;10:e135449 CrossRef Medline

12. Hernandez-Castillo CR, Vaca-Palomares I, Galvez V, et al. Cognitive deficits correlate with white matter deterioration in spinocerebellar ataxia type 2. J Int Neuropsychol Soc 2016;22:486-91 CrossRef Medline

13. Li H, Ma J, Zhang X. Diffusion tensor imaging of spinocerebellar ataxia type 12. Med Sci Monit 2014;20:1783-91 CrossRef Medline

14. Schmitz-Hübsch T, du Montcel ST, Baliko L, et al. Scale for the assessment and rating of ataxia: development of a new clinical scale. Neurology 2006;66:1717-20 CrossRef Medline

15. Weyer A, Abele M, Schmitz-Hübsch T, et al. Reliability and validity of the scale for the assessment and rating of ataxia: a study in $\mathbf{6 4}$ ataxia patients. Mov Disord 2007;22:1633-37 CrossRef Medline

16. Smith SM, Jenkinson M, Woolrich MW, et al. Advances in functional and structural MR image analysis and implementation as FSL. Neuroimage 2004;23:S208-19 CrossRef Medline

17. Smith SM, Jenkinson M, Johansen-Berg H, et al. Tract-based spatial statistics: voxelwise analysis of multi-subject diffusion data. Neuroimage 2006;31:1487-505 CrossRef Medline

18. Jenkinson M, Bannister P, Brady M, et al. Improved optimization for the robust and accurate linear registration and motion correction of brain images. Neuroimage 2002;17:825-41 CrossRef Medline

19. Nichols TE, Holmes AP. Nonparametric permutation tests for functional neuroimaging: a primer with examples. Hum Brain Mapp 2002;15:1-25 CrossRef Medline

20. Winkler AM, Ridgway GR, Webster MA, et al. Permutation inference for the general linear model. Neuroimage 2014;92:381-97 CrossRef Medline

21. Hernandez-Castillo CR, Galvez V, Diaz R, et al. Specific cerebellar and cortical degeneration correlates with ataxia severity in spinocerebellar ataxia type 7. Brain Imaging Behav 2016;10:252-57 CrossRef Medline

22. Renier LA, Anurova I, De Volder AG, et al. Preserved functional specialization for spatial processing in the middle occipital gyrus of the early blind. Neuron 2010;68:138 - 48 CrossRef Medline

23. Martínez A, Anllo-Vento L, Sereno MI, et al. Involvement of striate and extrastriate visual cortical areas in spatial attention. $\mathrm{Nat} \mathrm{Neu-}$ rosci 1999;2:364-69 CrossRef Medline

24. Hernandez-Castillo CR, Alcauter S, Galvez V, et al. Disruption of visual and motor connectivity in spinocerebellar ataxia type 7. Mov Disord 2013;28:1708-16 CrossRef Medline

25. Hernandez-Castillo CR, Galvez V, Morgado-Valle C, et al. Wholebrain connectivity analysis and classification of spinocerebellar ataxia type 7 by functional MRI. Cerebellum Ataxias 2014;1:2 CrossRef Medline

26. Schmahmann JD. Disorders of the cerebellum: ataxia, dysmetria of thought, and the cerebellar cognitive affective syndrome. J Neuropsychiatry Clin Neurosci 2004;16:367-78 CrossRef Medline

27. Cosottini M, Giannelli M, Siciliano G, et al. Diffusion-tensor MR imaging of corticospinal tract in amyotrophic lateral sclerosis and progressive muscular atrophy. Radiology 2005;237:258-64 CrossRef Medline

28. Pierpaoli C, Barnett A, Pajevic S, et al. Water diffusion changes in Wallerian degeneration and their dependence on white matter architecture. Neuroimage 2001;13(6 pt 1):1174-85 CrossRef Medline

29. Mandelli ML, De Simone T, Minati L, et al. Diffusion tensor imaging of spinocerebellar ataxias types 1 and 2. AJNR Am J Neuroradiol 2007;28:1996-2000 CrossRef Medline

30. Vos SB, Jones DK, Jeurissen B, et al. The influence of complex white matter architecture on the mean diffusivity in diffusion tensor MRI of the human brain. Neuroimage 2012;59:2208-16 CrossRef Medline 